\title{
RESOLVING THE LARGE-SCALE SPECTRAL VARIABILITY OF THE LUMINOUS SEYFERT 1 GALAXY 1H 0419-577: EVIDENCE FOR A NEW EMISSION COMPONENT AND ABSORPTION BY COLD DENSE MATTER
}

\author{
K. A. Pounds, ${ }^{1}$ J. N. Reeves, ${ }^{2,3}$ K. L. Page, ${ }^{1}$ And P. T. O’Brien ${ }^{1}$ \\ Received 2004 May 17; accepted 2004 August 3
}

\begin{abstract}
An XMM-Newton observation of the luminous Seyfert 1 galaxy 1H 0419-577 in 2002 September, when the source was in an extreme low-flux state, found a very hard X-ray spectrum at $1-10 \mathrm{keV}$ with a strong soft excess below $\sim 1 \mathrm{keV}$. Comparison with an earlier XMM-Newton observation when 1H 0419-577 was "X-ray bright" indicated that the dominant spectral variability was due to a steep power law or cool Comptonized thermal emission. Four further XMM-Newton observations, with 1H 0419-577 in intermediate-flux states, now support that conclusion, while we also find the variable emission component in intermediate-state difference spectra to be strongly modified by absorption in low-ionization matter. The variable "soft excess" then appears to be an artifact of absorption of the underlying continuum, while the "core" soft emission can be attributed to recombination in an extended region of more highly ionized gas. We note the wider implications of finding substantial cold dense matter overlying (or embedded in) the X-ray continuum source in a luminous Seyfert 1 galaxy.
\end{abstract}

Subject headings: galaxies: individual (1H 0419-577, LB 1727) — galaxies: Seyfert — X-rays: galaxies

Online material: color figures

\section{INTRODUCTION}

1H $0419-577$ (also known as LB 1727) is a radio-quiet (8.4 GHz flux $\sim 3$ mJy; Brissenden et al. 1987) Seyfert galaxy at a redshift $z \sim 0.104$. Optical spectra from the Anglo-Australian Telescope (AAT; Turner et al. 1999) and ESO (Guainazzi et al. 1998) showed 1H 0419-577 to be a typical broad-line Seyfert 1 with a strong big blue bump (BBB). It has been widely studied at X-ray energies and was found to exhibit an unusual degree of spectral variability. A short pn camera observation early in the XMM-Newton program reported a "typical" Seyfert $1 \mathrm{X}$-ray spectrum with a power law of photon index $\Gamma \sim 1.9$ together with a strong soft excess (Page et al. 2002). Using the $\mathrm{H} \beta$ line width from Grupe et al. (2004) and the relationship with black hole mass of Kaspi et al. (2000), we estimate a supermassive black hole (SMBH) of $1.3 \times 10^{8} M_{\odot}$. The [O III] $5007 \AA$ line width measure of the stellar velocity dispersion (Nelson 2000) gives a very similar figure.

To improve the X-ray data on $1 \mathrm{H} 0419-577$ a new series of five XMM-Newton observations was carried out at approximately 3 month intervals over the period 2002 September to 2003 September. The first of those new observations, when $1 \mathrm{H} 0419-577$ was found to be in an extremely faint state, has been reported in Pounds et al. (2004b, hereafter Paper I). Three points of particular note resulting from this first new $X M M$ Newton observation of $1 \mathrm{H} 0419-577$ were that (1) an unusually hard (flat) power-law fit $(\Gamma \sim 1.0)$ to the EPIC data over the 2-10 keV band also exhibited curvature indicative of an extreme relativistic Fe K emission line or partial covering of the continuum by a substantial column of "cold" gas; (2) although the XMM-Newton RGS features were faint, the unambiguous detection of emission lines of $\mathrm{O}$ VII and $\mathrm{O}$ VIII provided clear evidence for an extended region of photoionized gas in the

\footnotetext{
1 Department of Physics and Astronomy, University of Leicester, Leicester LE1 7RH, UK; kap@star.le.ac.uk.

2 Laboratory for High Energy Astrophysics, Code 662, NASA Goddard Space Flight Center, Greenbelt, MD 20771.

${ }^{3}$ Universities Space Research Association.
}

nucleus of $1 \mathrm{H}$ 0419-577; and (3) a comparison of the raw EPIC data with data obtained in 2000 December, when 1H 0419-577 was considerably brighter in the X-ray band, showed that the large-scale spectral variability in $1 \mathrm{H} 0419-577$ could be described by a variable, steep $(\Gamma \sim 2.5)$ power-law component.

In the present paper we analyze the remaining four observations from the new XMM-Newton campaign (in 2002 December and 2003 March, June, and September), which together explore the X-ray spectrum of $1 \mathrm{H} 0419-577$ over a range of flux states lying between the extremes of 2000 December and 2002 September.

\section{OBSERVATIONS AND X-RAY DATA}

The new observations took place on 2002 December 27 (orbit 558) and 2003 March 30 (orbit 605), June 25 (orbit 649) and September 16 (orbit 690), with on-target exposures of $\sim 10.2$, $\sim 13.9, \sim 13.1$, and $\sim 13.9 \mathrm{ks}$, respectively. X-ray data were available throughout each observation from the EPIC pn (Strüder et al. 2001) and MOS (Turner et al. 2001) cameras and the RGS (den Herder et al. 2001). These new data add to the earlier XMMNewton observations of 1H 0419-577 on 2000 December 4 (orbit 181) and 2002 September 5 (orbit 512), when the source was found to be in historically high- and low-flux states, respectively. Those earlier observations have been reported in Page et al. (2002) and in Paper I.

EPIC data were initially screened with the XMM-Newton SAS version 5.4 software, and events corresponding to patterns 0-4 (single- and double-pixel events) were selected for the pn data and patterns $0-12$ were selected for MOS1 and MOS2, the latter then being combined. A low-energy cut of $300 \mathrm{eV}$ was applied to all EPIC data, and known hot or bad pixels were removed. Source counts were obtained from a circular region of $45^{\prime \prime}$ radius centered on $1 \mathrm{H} 0419-577$, with the background being taken from a similar region offset from, but close to, the source. The X-ray light curve of $1 \mathrm{H} 0419-577$ was essentially flat throughout each observation, and the background rate was low (except in orbit 558, when noisy data were edited out). We therefore integrated each data set for spectral analysis. 


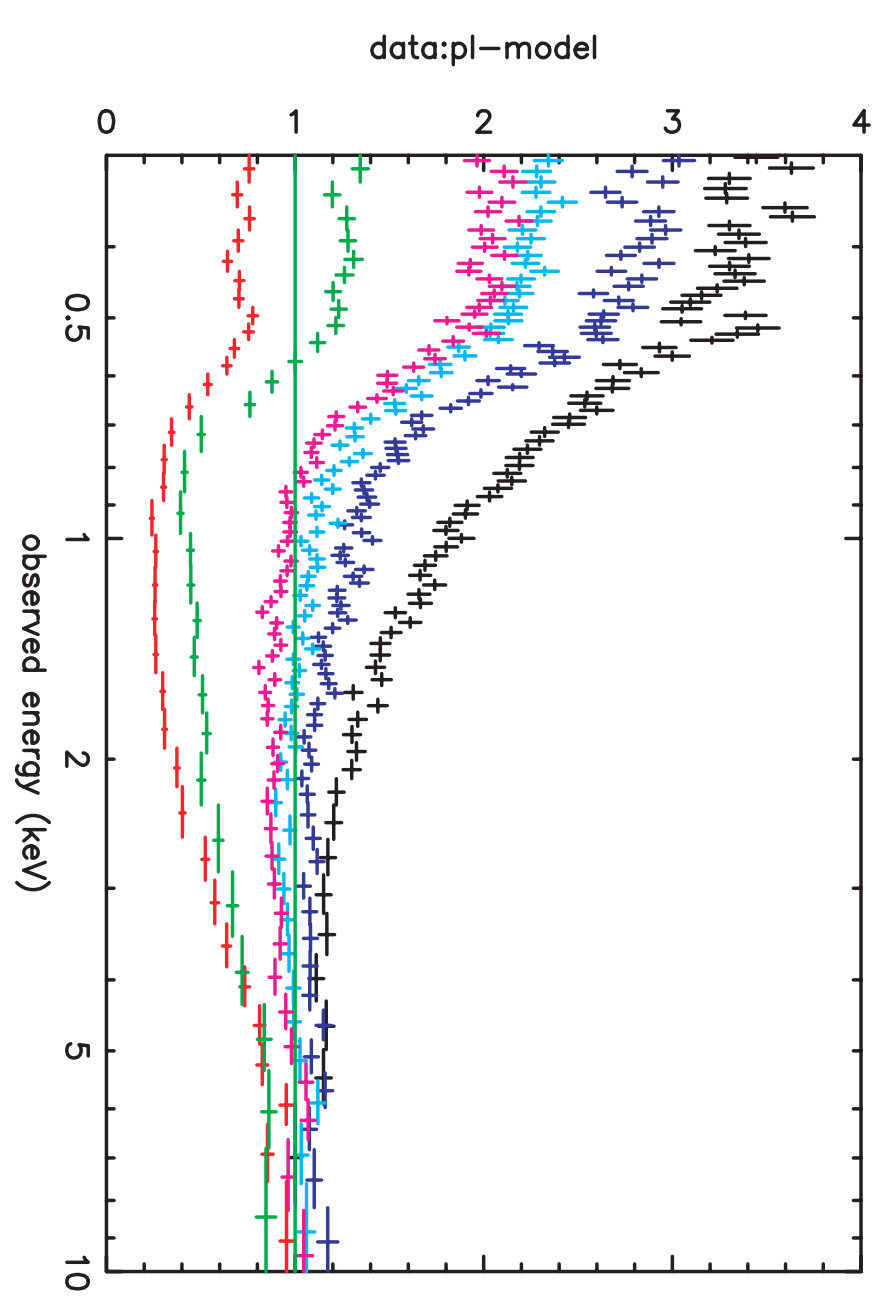

FIG. 1.-EPIC spectral data from the observations of 2000 December, orbit 181 (black); 2002 September, orbit 512 (red); 2002 December, orbit 558 (green); 2003 March, orbit 605 (purple); 2003 June, orbit 649 (light blue); and 2003 September, orbit 690 (magenta); compared (at 2-10 keV) with a power law of photon index $\Gamma=1.73$. For clarity, only the pn camera data are shown.

Individual EPIC spectra were binned to a minimum of 100 counts per energy bin to facilitate the use of the $\chi^{2}$ minimization technique in spectral fitting and ensure adequate statistics in the 5-10 keV band. Spectral fitting was based on the XSPEC package (Arnaud 1996), and all fits included absorption due to the line-of-sight Galactic column $N_{\mathrm{H}}=2 \times 10^{20} \mathrm{~cm}^{-2}$. Errors are quoted at the $90 \%$ confidence level $\left(\Delta \chi^{2}=2.7\right.$ for one interesting parameter).

We were fortunate to observe $1 \mathrm{H} 0419-577$ in widely differing flux states, ranging conveniently between the low- and high-flux state extremes reported previously. Figure 1 shows the background-subtracted ( $p$ n camera) spectral data for all six observations, compared with a mean power law fit (at 2$10 \mathrm{keV}$ ) of $\Gamma=1.73$. The plot shows very clearly that the largest flux variation occurs in the soft X-ray band, with all spectra converging above $\sim 5 \mathrm{keV}$. The only significance we would claim for the mean power-law fit is in the spectral index being close to the canonical value for Seyfert 1 galaxies (for fits excluding reflection; Nandra \& Pounds 1994), suggesting that this unusually variable active galactic nucleus (AGN) has an average X-ray spectrum typical of the class. Retaining our previous descriptions of the orbit 181 and 512 data as highand low-flux states, respectively, we now define orbit 558 as
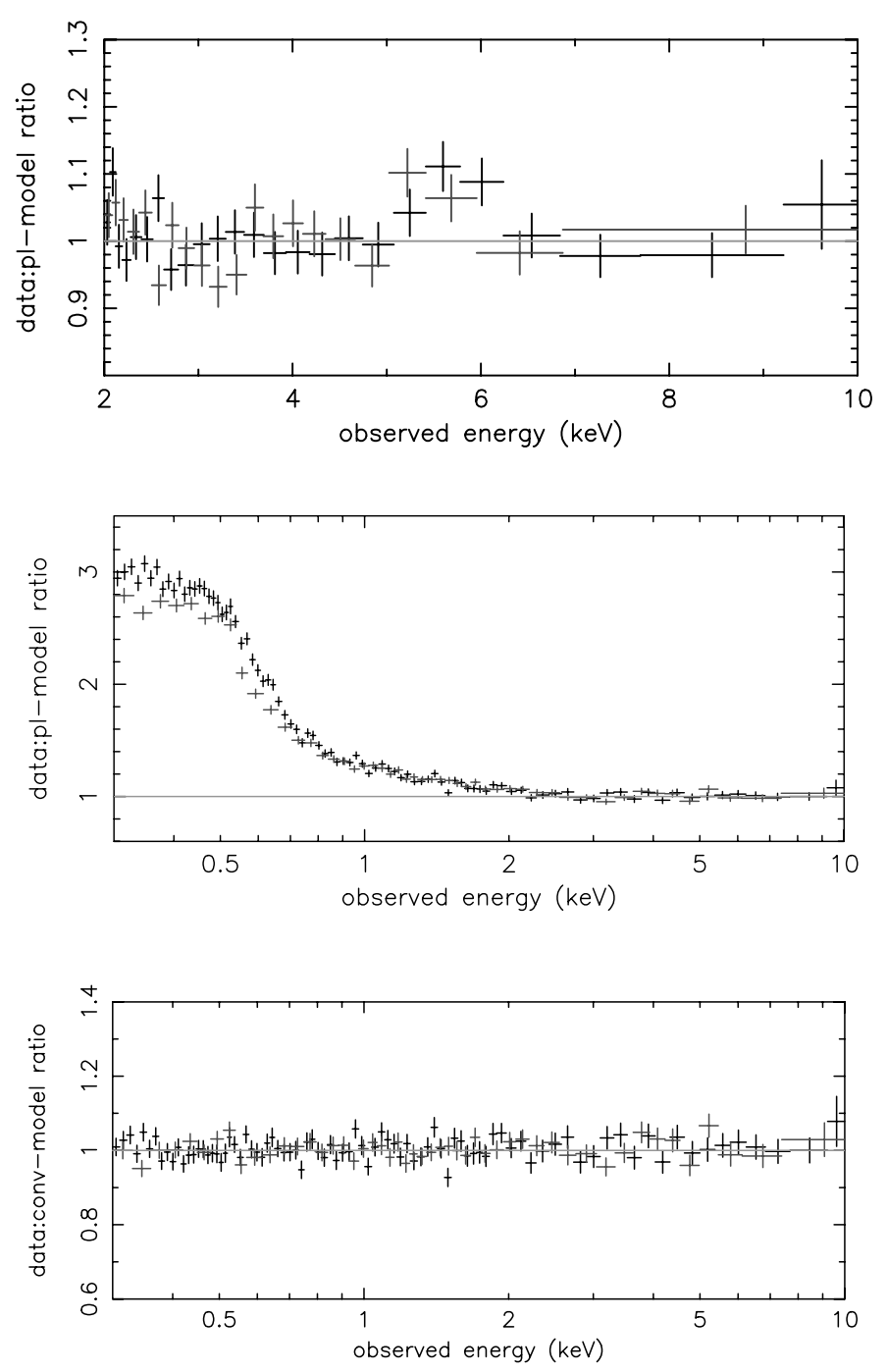

FIG. 2.-Top: Ratio of pn (black) and MOS data (gray) to 2-10 keV powerlaw fits to the intermediate-flux state data (2003 June/September), showing a weak Fe K emission line. Middle: Extrapolation of the $2-10 \mathrm{keV}$ power law to $0.3 \mathrm{keV}$, showing a strong soft excess. Bottom: Ratio of data to the conventional multicomponent model spectrum described in $\S 3$. [See the electronic edition of the Journal for a color version of this figure.]

"mid-low," orbit 605 as "mid-high," and group orbits 649 and 690 together to give a well-defined "intermediate" flux state spectrum.

In the following analysis spectral parameters are quoted in the rest frame of 1H 0419-577, while figures (except Fig. 10) retain the observed photon energy scale. In general, we have fitted the pn and MOS data simultaneously, with only the powerlaw parameters untied, reflecting the well-known systematic difference in deduced continuum slopes.

\section{SPECTRAL FITTING OF THE INTERMEDIATE-FLUX STATE EPIC DATA}

We began the EPIC spectral analysis in the conventional way by fitting a power law over the hard X-ray $(2-10 \mathrm{keV})$ band, aiming thereby to minimize the effects of soft X-ray emission and/or low-energy absorption. This fit yielded a photon index of $\Gamma=1.60 \pm 0.01$ (pn) and $\Gamma=1.55 \pm 0.02$ (MOS). Statistically, the simple power-law fit was quite good, with $\chi^{2}$ of 393 for 357 degrees of freedom (dof). The most obvious residual spectral feature is a weak emission line observed just below $\sim 6 \mathrm{keV}$ (Fig. 2, top). The addition of a Gaussian emission line 


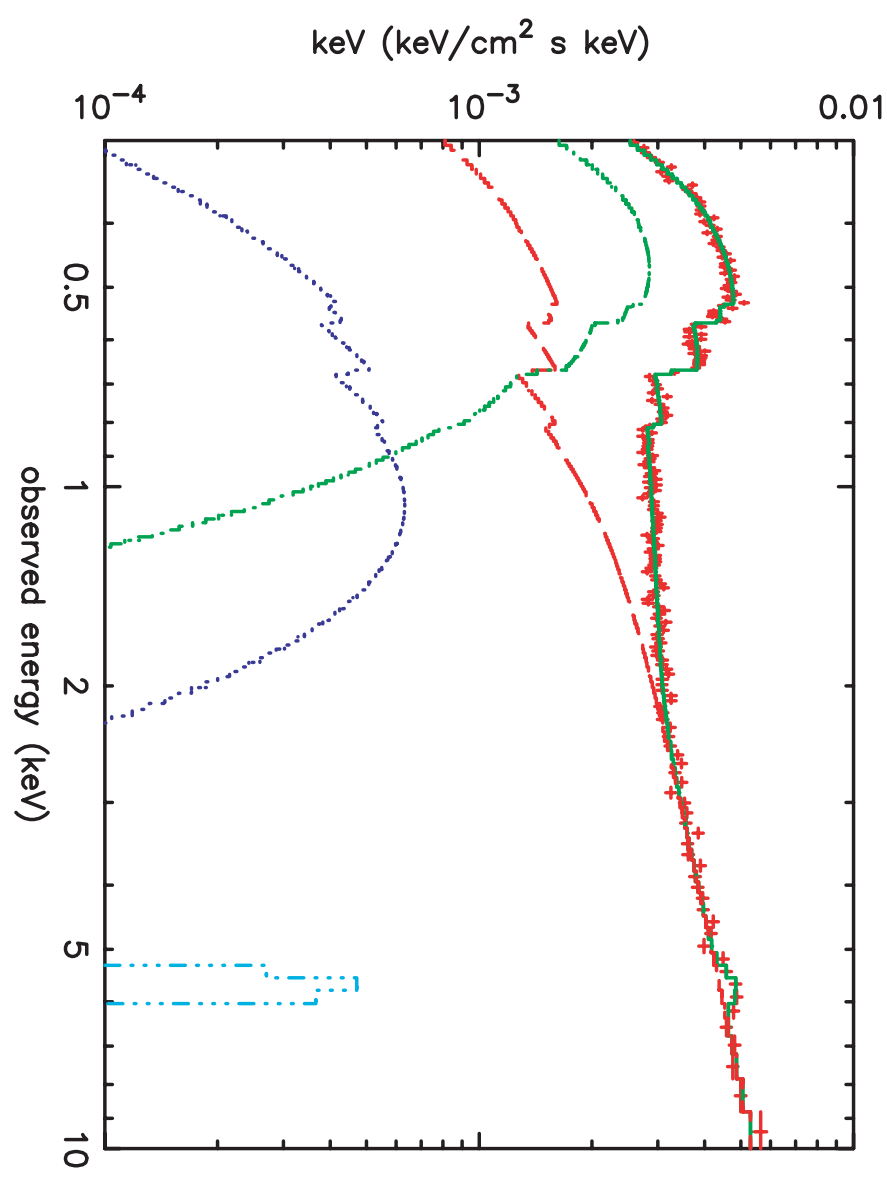

FIg. 3.- Unfolded broadband spectrum fitted to the intermediate-flux state data for $1 \mathrm{H} 0419-577$. The spectral components of this conventional fit are: power law (red), blackbodies ( purple and green), and Fe K emission line (light blue). For clarity, only the pn data are shown.

improved the fit (to $\chi^{2}=373 / 353$ dof), with a line energy (in the AGN rest frame) of $6.25 \pm 0.12 \mathrm{keV}$, rms width $\sigma=$ $320 \pm 150 \mathrm{eV}$, flux $=(1.5 \pm 0.6) \times 10^{-5}$ photons $\mathrm{s}^{-1} \mathrm{~cm}^{-2}$, and $\mathrm{EW}=100 \pm 40 \mathrm{eV}$.

Extending this spectral fit to $0.3 \mathrm{keV}$ revealed the strong excess below $\sim 1 \mathrm{keV}$ (Fig. 2, middle). The addition of blackbody components of $k T \sim 110$ and $\sim 250 \mathrm{eV}$ modeled the soft excess quite well, but it was necessary to add (ad hoc) absorption edges at $\sim 0.62 \mathrm{keV}(\tau \sim 0.22), \sim 0.74 \mathrm{keV}(\tau \sim 0.26)$, and $\sim 0.90 \mathrm{keV}(\tau \sim 0.10)$ to achieve a statistically acceptable fit $\left(\chi^{2}=825 / 783\right.$ dof $)$. Figure 3 reproduces this "conventional" fit to the intermediate-flux level data for $1 \mathrm{H} 0419-577$, which we note has model parameters typical for a luminous Seyfert 1 galaxy.

The above spectral fitting allowed the mean X-ray fluxes of the intermediate-state spectrum of $1 \mathrm{H} 0419-577$ to be determined. These were $5.9 \times 10^{-12}$ ergs s${ }^{-1} \mathrm{~cm}^{-2}(0.3-1 \mathrm{keV}), 3.0 \times$ $10^{-12} \mathrm{ergs} \mathrm{s}^{-1} \mathrm{~cm}^{-2}(1-2 \mathrm{keV})$, and $1.05 \times 10^{-11} \mathrm{ergs} \mathrm{s}^{-1} \mathrm{~cm}^{-2}$ $(2-10 \mathrm{keV})$. Combining these fluxes yields an intermediatestate $0.3-10 \mathrm{keV}$ luminosity for $1 \mathrm{H} 0419-577$ of $4.3 \times 10^{44} \mathrm{ergs}$ $\mathrm{s}^{-1}\left(H_{0}=75 \mathrm{~km} \mathrm{~s}^{-1} \mathrm{Mpc}^{-1}\right)$.

\section{AN ALTERNATIVE SPECTRAL FIT \\ TO THE INTERMEDIATE-FLUX STATE SPECTRUM}

Our main aim in the present study is to better understand the large-scale spectral variability of 1H 0419-577, and thereby of AGNs more generally. In Paper I we found that the difference
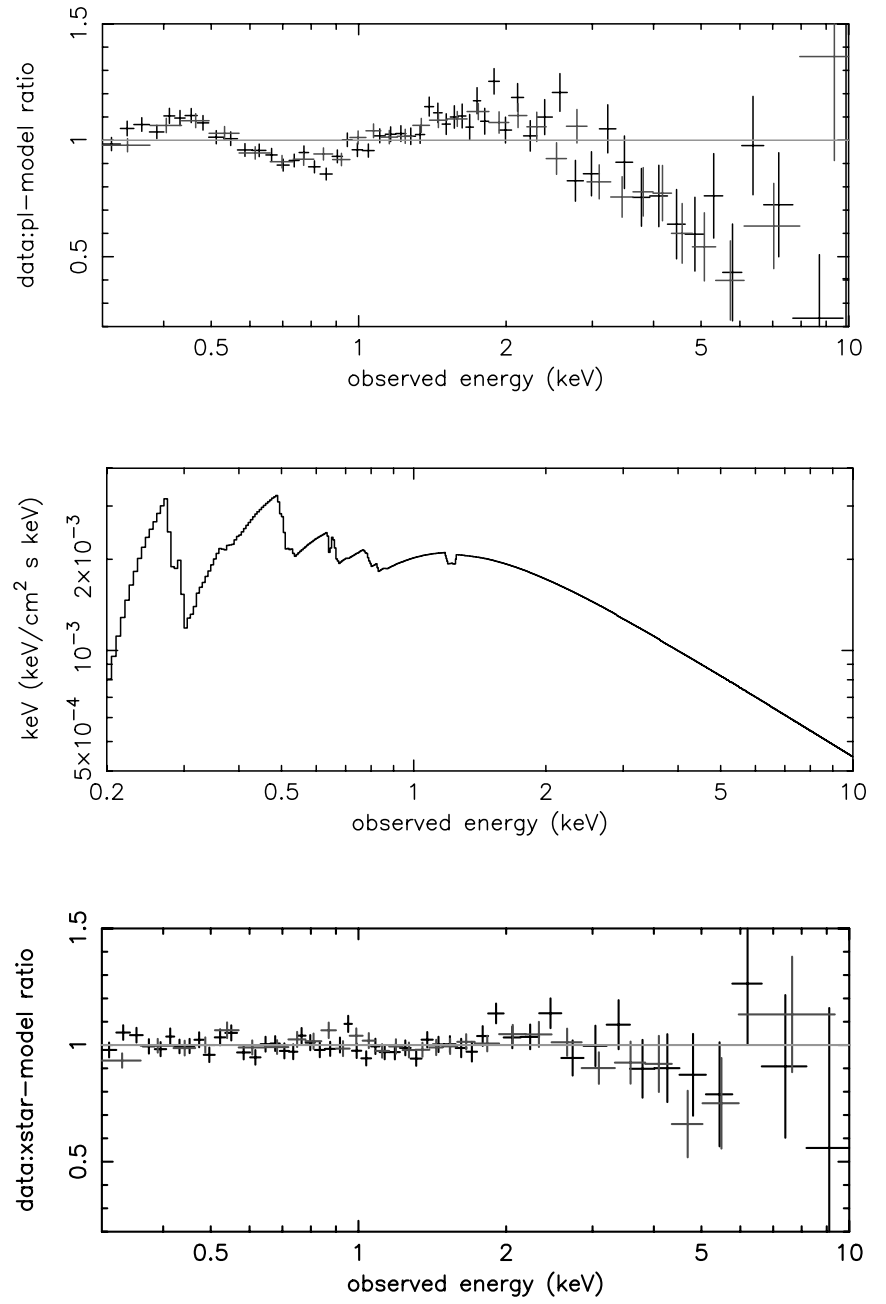

FIG. 4.-Top: Ratio of data to a single power-law fit for the intermediatestate difference spectrum. Middle: Power law plus XSTAR absorption model for the same difference spectrum, with strong absorption edge structure (in order of increasing energy) of $\mathrm{C}, \mathrm{O}, \mathrm{Fe}$, and Ne. Bottom: Ratio of difference spectrum data to the power law plus absorption model in XSTAR. See $\S 4$ for details. The small spectral feature seen close to the Mg edge at $\sim 1.2 \mathrm{keV}$ is due to the curtailment of the tabulated photoionization cross sections. [See the electronic edition of the Journal for color versions of parts of this figure.]

spectrum between high- and low-state EPIC pn data (the highflux difference spectrum) could be well fitted by a power law of $\Gamma \sim 2.5$, steepening to $\sim 2.7$ above $\sim 2 \mathrm{keV}$. This simple fit suggested that the low-state spectrum (hard power law plus soft excess) might represent a nonvarying core emission, leaving the main spectral change to be represented by the variable-flux power-law component. To further explore that possibility, we now reanalyze the new intermediate-flux state EPIC data, after subtracting the 2002 September data, to yield the intermediatestate difference spectrum.

We find that the resulting intermediate-state difference spectrum is similar to the high-state difference spectrum, with a mean $0.3-10 \mathrm{keV}$ power-law index of $\Gamma \sim 2.41$ (pn), steepening to $\sim 2.85$ when fitted above $\sim 2 \mathrm{keV}$. However, the fit is much less good $\left(\chi^{2}=1174 / 799\right.$ dof $)$, owing to a broad deficit of flux at $\sim 0.5-1 \mathrm{keV}$ (Fig. 4 , top). If the large-scale spectral variability in $1 \mathrm{H} 0419-577$ is indeed well modeled by a steep, variable-flux power law, as proposed in our previous analysis of the high-state difference spectrum (Paper I), the new data suggest that the variable emission component is modified by absorption in intermediate-flux states. To model that possibility 
we then compared the intermediate-state difference spectrum with a power law plus a photoionized absorber, represented by XSTAR (Kallman et al. 1996). Here the absorption is compared with a grid of ionized absorbers, with column density, ionization parameter $\xi\left(\xi=L / n r^{2}\right.$, where $n$ is the gas density at a distance $r$ from the ionizing source of luminosity $L$ ), and outflow (or inflow) velocity as variable parameters. All abundant elements from $\mathrm{C}$ to $\mathrm{Fe}$ are included, with the relative abundances as further variable parameters. The resulting fit was good ( $\chi^{2}$ of 839/790), with a column density $N_{\mathrm{H}} \sim 4 \times 10^{21} \mathrm{~cm}^{-2}$ of low-ionization gas $(\log \xi=-1.7 \pm 0.4)$. The relative abundances of the key elements, of $\mathrm{C}, \mathrm{N}, \mathrm{O}, \mathrm{Ne}, \mathrm{Mg}$, and Fe, were $0.7,0.9,0.20,0.75,1.0$, and 1.0, although only $\mathrm{O}, \mathrm{Ne}$, and $\mathrm{Fe}$ were well constrained in the fit. This best fit was obtained with a redshift corresponding to $1 \mathrm{H} 0419-577$, implying that the substantial column of cold absorbing gas is local to $1 \mathrm{H} 0419-$ 577. The power-law slopes in the fit increased to $\Gamma \sim 2.8(\mathrm{pn})$ and $\Gamma \sim 2.7$ (MOS), while still leaving a further spectral steepening above $\sim 3 \mathrm{keV}$ (Fig. 4, bottom). Figure 4 (middle) illustrates the relevant XSTAR model, in which the absorption structure is dominated by continuum absorption, in increasing photon energy, of $\mathrm{C}, \mathrm{O}, \mathrm{Fe}$, and $\mathrm{Ne}$.

\subsection{The Form of the Variable Emission Component}

In the above spectral fit we assumed that the variable emission component in 1H $0419-577$ has the form of a power law. A similar conclusion was proposed from an $A S C A$ study of the Seyfert 1 galaxy MCG -6-30-15 (Shih et al. 2002) and supported by extended XMM-Newton observations of the same source (Fabian \& Vaughan 2003). However, it is notable that for 1H 0419-577 the power-law fit including absorption (Fig. 4, bottom) indicates further steepening above $\sim 3 \mathrm{keV}$, and we recall that the compTT model (Titarchuk 1994) gave an even better fit to the high-state difference spectrum reported in $\mathrm{Pa}$ per I. Figure 5 reproduces the residuals to the single powerlaw and compTT fits to the high-state difference spectrum. The thermal Comptonization model has the additional appeal of being more physical than a power-law fit. Since the intrinsic curvature of the thermal continuum might significantly affect the derived absorption parameters, we therefore repeated the analysis of the intermediate-state difference spectrum with a model involving the emergence of a cool Comptonized emission component modified by absorption in ionized matter.

Replacing the power law of $\S 4$ with a Comptonized emission component with an initial temperature of $k T \sim 2.7 \mathrm{keV}$ and optical depth $\sim 4.4$, as found for the high-flux difference spectrum, the fit was indeed better than for the power-law model, though still poor $\left(\chi^{2}=1102 / 796\right.$ dof $)$, with data: model residuals similar to those for the power-law fit in Figure 4 (top). Photoionized absorption represented by XSTAR was then added to the model. The outcome was a very good fit $\left(\chi^{2}=\right.$ $828 / 784$ dof ) for a Comptonized emission component of $k T=$ $2.3 \pm 0.4 \mathrm{keV}$ and optical depth $4.5 \pm 0.7$. The absorption was again well modeled by low-ionization matter, with a column density of $N_{\mathrm{H}} \sim 4.4 \times 10^{21} \mathrm{~cm}^{-2}$ and an ionization parameter of $\log \xi=-1.8 \pm 0.3$. The relative abundances of the key elements of C, $\mathrm{N}, \mathrm{O}, \mathrm{Ne}, \mathrm{Mg}$, and $\mathrm{Fe}$, were $0.2,0.2,0.12,0.4,0.7$, and 0.66 , respectively, with the lower abundances of $\mathrm{C}$ and $\mathrm{N}$ (compared with the power-law fit) adjusting to the low-energy curvature in the continuum fit.

Figure 6 (top) shows the best-fit Comptonized emission component and pn camera data for the intermediate-flux difference spectrum, with the XSTAR absorption component removed.
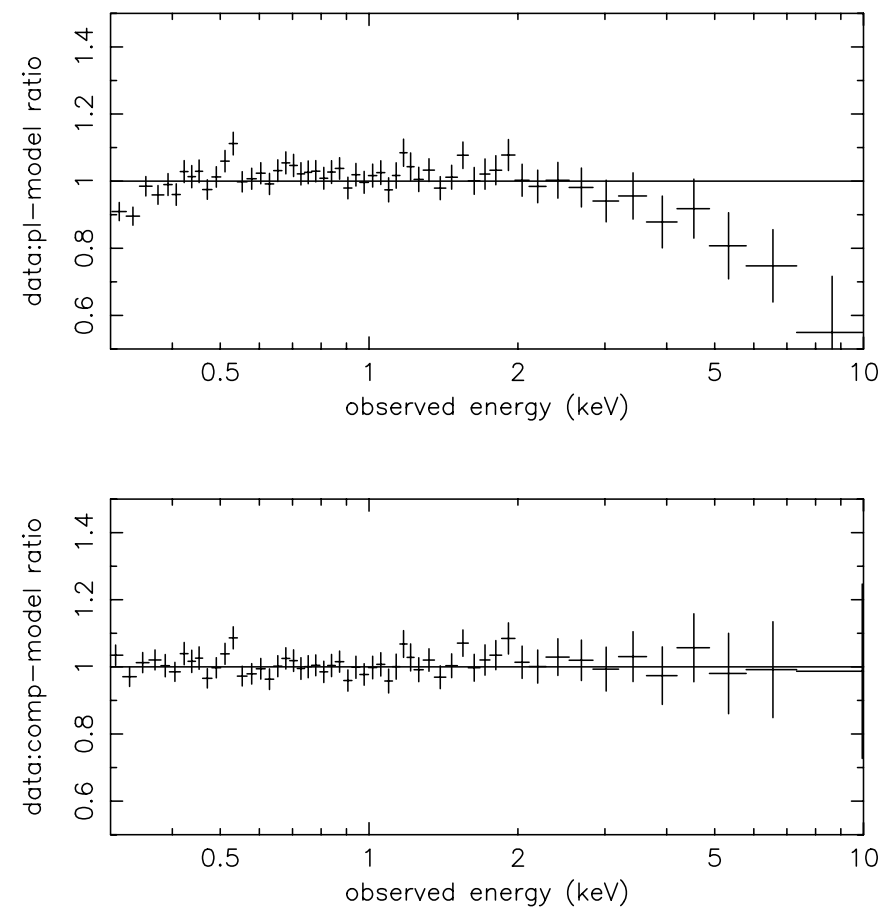

FIG. 5.-Top: Ratio of high-state difference spectrum (2000 December) to a single power-law model of photon index 2.47. Bottom: Ratio of same data to a thermal Comptonization model, with seed photons of $k T=73 \pm 3 \mathrm{eV}$ scattered in a plasma of temperature $k T=2.7 \pm 0.6 \mathrm{keV}$ and optical depth $\tau=4.4 \pm 0.6$.

Figure 6 (middle) reproduces the model spectrum, while Figure 6 (bottom) illustrates the quality of the resulting fit.

\section{SPECTRAL FITS TO THE MID-LOW-FLUX STATE SPECTRUM}

Taken together with the high-state difference spectrum reported in Paper I, the above analysis of the intermediate-state difference spectrum suggests that the large-scale spectral variability of $1 \mathrm{H} 0419-577$ is indeed due to an emerging emission component that, at intermediate-flux levels, bears the imprint of absorption by low ionization matter. To see if that trend of variable absorption with flux level is continuous, we repeated the above analysis for the mid-low-flux state observation, but again starting with a conventional power law plus blackbody fit.

Fitting a power law over the hard X-ray $(2-10 \mathrm{keV})$ band yielded a photon index of $\Gamma=1.41 \pm 0.03$ (pn) and $\Gamma=$ $1.35 \pm 0.06$ (MOS) for the mid-low-flux spectrum. Extending this fit to $0.3 \mathrm{keV}$ showed a soft excess rising sharply below $\sim 0.7 \mathrm{keV}$ (Fig. 7, top). The narrower profile of this soft excess (compared with that in Fig. 2, top) allowed it to be well modeled by the addition of a single blackbody component of $k T \sim 102 \mathrm{eV}$, no hotter blackbody being required. In this case a single absorption edge $(\tau \sim 0.8$ at $0.76 \pm 0.01 \mathrm{keV})$ was needed to complete an excellent fit $\left(\chi^{2}=245 / 244\right.$ dof $)$. Figure 7 (middle) shows the ratio of data to this power-law, blackbody, and absorption edge model. In summary, a conventional fit to the mid-low-flux spectrum of $1 \mathrm{H} 0419-577$ shows a hard (flat) power law with a sharply rising (cool) soft excess and a deep absorption edge at $\sim 0.76 \mathrm{keV}$ (in the AGN rest frame).

The above fit provided a measure of the $\mathrm{X}$-ray fluxes and luminosity of $1 \mathrm{H} 0419-577$ in the mid-low-flux state, which 

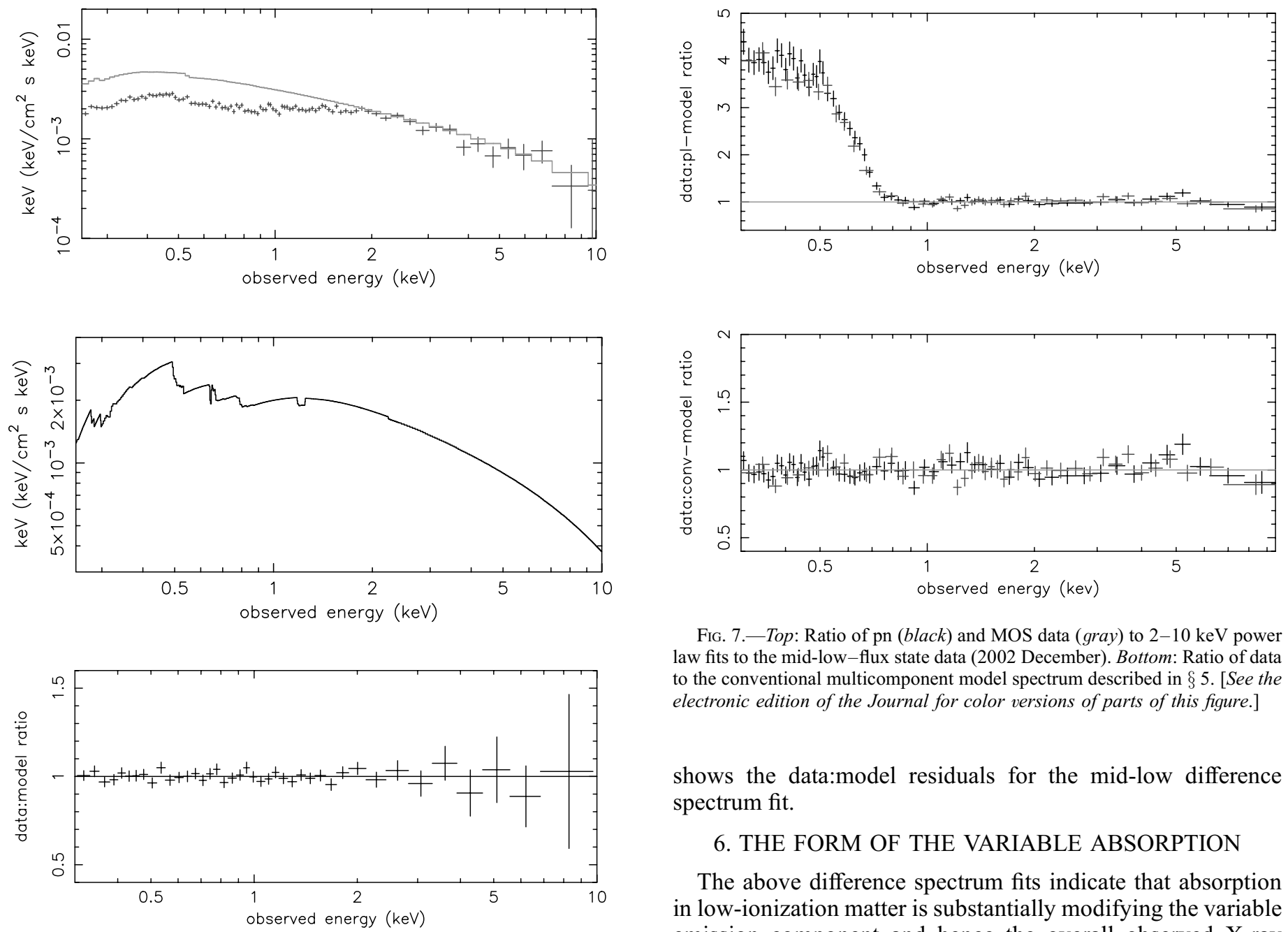

FIG. 7.-Top: Ratio of pn (black) and MOS data (gray) to $2-10 \mathrm{keV}$ power law fits to the mid-low-flux state data (2002 December). Bottom: Ratio of data to the conventional multicomponent model spectrum described in $\S 5$. [See the electronic edition of the Journal for color versions of parts of this figure.]

shows the data:model residuals for the mid-low difference spectrum fit.

\section{THE FORM OF THE VARIABLE ABSORPTION}

The above difference spectrum fits indicate that absorption in low-ionization matter is substantially modifying the variable emission component and hence the overall observed X-ray spectrum of $1 \mathrm{H} 0419-577$; furthermore, we find the absorbing matter to become more ionized and the column density to fall as the continuum flux rises. To further explore the variable absorber we then computed the ratio of the "raw" intermediateand mid-low-flux spectra. The shape of that ratio plot (Fig. 9, top) is particularly interesting, the marked drop observed at $\sim 0.7 \mathrm{keV}$ coinciding with low-ionization $\mathrm{Fe}$ absorption edges, with no obvious change in the flux ratio at the corresponding edges of low-ionization oxygen. The flux ratio plot is thus consistent with the specific XSTAR models that match the relatively deeper absorption edge near $0.7 \mathrm{keV}$ with the lower ionization parameter for the lower flux state spectrum (cf. Figs. 6, middle, and 8 , middle). A weaker feature at $\sim 0.9 \mathrm{keV}$ in Figure 9 (top), close to the absorption edge structure of $\mathrm{Ne}$, is also qualitatively consistent with the individual XSTAR fits. Figure 9 (bottom) shows the ratio of the mid-high- to high-flux state spectra, indicating that the same pattern of decreasing absorption continues as the flux level of $1 \mathrm{H} 0419-577$ rises toward the high state.

The different profile of the absorption in the intermediateand mid-low-state difference spectral fits is determined by the changing ionization parameter. To better understand that change we replot the key section of each XSTAR model fit in Figure 10, where for ease of comparison with listed edges the energy axes are adjusted to the rest frame of $1 \mathrm{H} \mathrm{0419-577}$ and the principal absorbing ion stages are noted against the respective absorption features. Reference to the detailed absorption cross sections for ground states of $\mathrm{O}$ and $\mathrm{Fe}$ (Kallman 

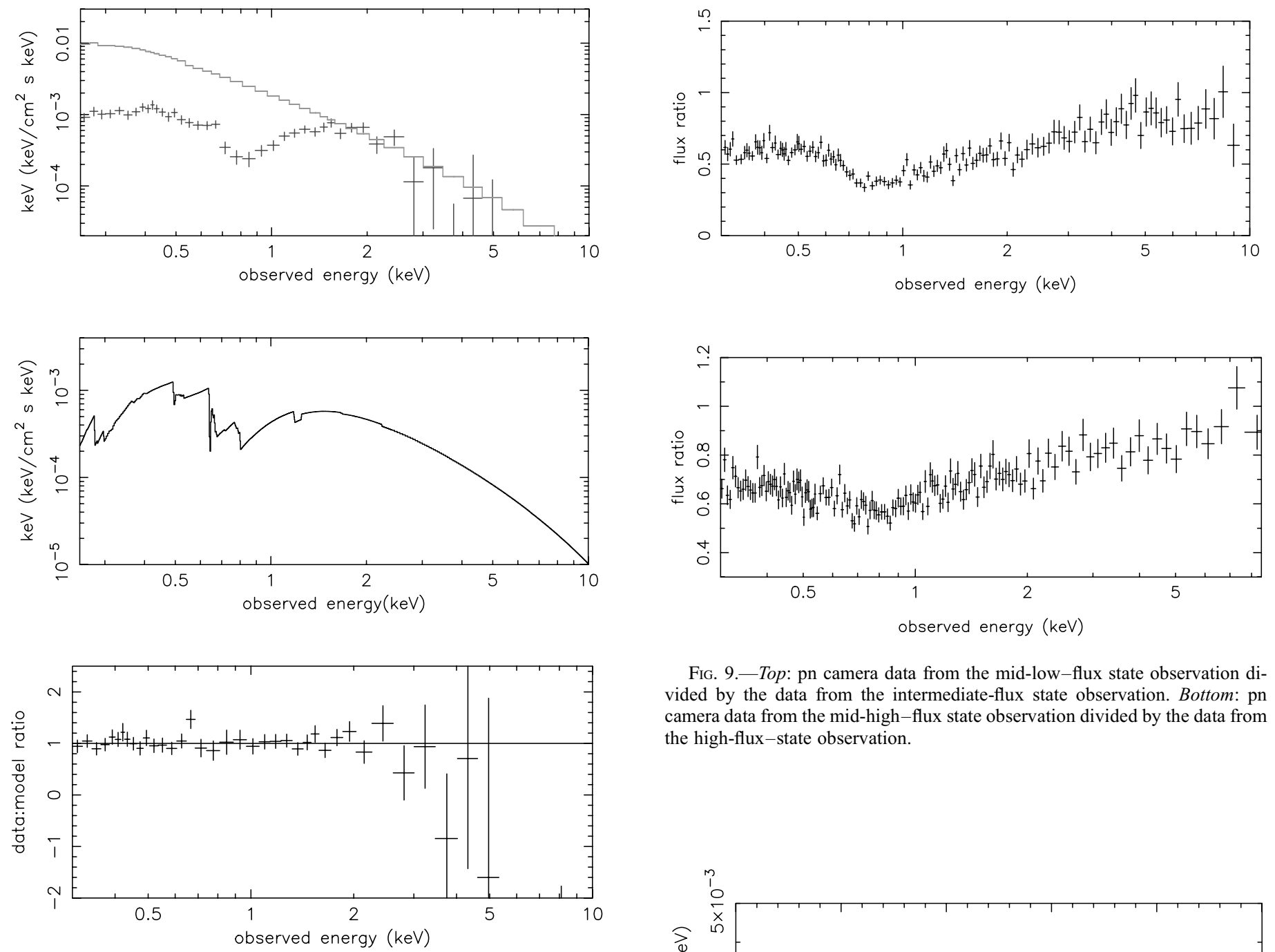

Fig. 8.-Top: Comptonized emission component and pn camera data for the mid-low-state difference spectrum of 1H 0419-577. Middle: Comptonized emission plus XSTAR absorption model for the mid-low-state difference spectrum. Bottom: Data to model ratio. [See the electronic edition of the Journal for a color version of part of this figure.]

\& Bautista 2001) then provides a qualitative explanation for the differential absorption we see in $1 \mathrm{H} 0419-577$, since whereas the threshold energy cross section in $\mathrm{Fe}$ increases by a factor $\sim 2.5$ from $\mathrm{Fe} v$ to $\mathrm{Fe}$ I, the threshold cross sections increase by only $\sim 40 \%$ from $\mathrm{O}$ IV to $\mathrm{O}$ I.

In summary, we find that the emerging emission component responsible for the main spectral change in $1 \mathrm{H} 0419-577$ is modified by low-energy absorption in a substantial column density of low-ionization matter. Furthermore, while remaining low the ionization state of the absorber increases as $1 \mathrm{H} 0419-$ 577 gets brighter, while the absorbing column (or perhaps the covering factor) simultaneously decreases, and the increasing ionization parameter provides a natural explanation of the changing energy profile of the absorption trough.

\section{SPECTRAL LINES IN THE RGS DATA}

Given the above evidence for substantial absorbing matter modifying the EPIC spectrum, it is of obvious importance to check whether this is consistent with the higher resolution RGS data. To pursue that question we examined the simultaneous
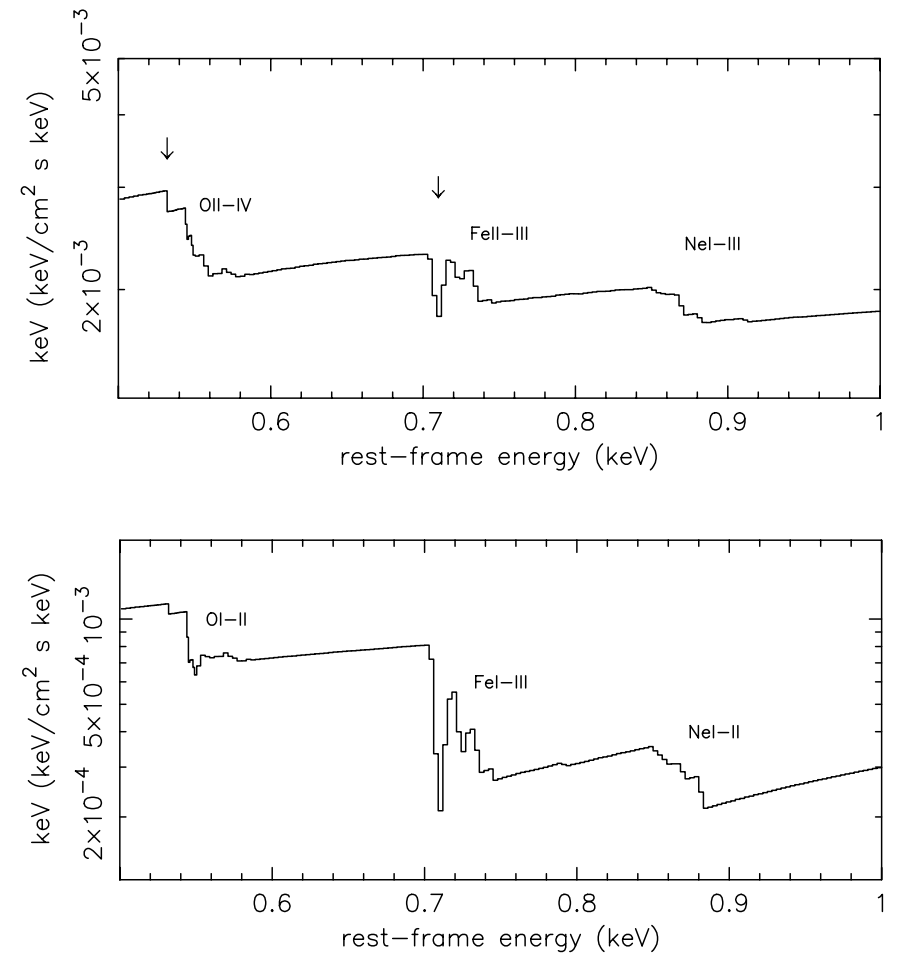

FIG. 10.-Top: Section of the compTT and XSTAR model fitted to the intermediate-state difference spectrum showing the absorption edge structure in $\mathrm{O}, \mathrm{Fe}$, and $\mathrm{Ne}$. The arrows note the $\mathrm{O}$ I edge due to the interstellar column in the line of sight to $1 \mathrm{H} 0419-577$ and the Fe 2-3 UTA. Bottom: Same plot for the corresponding fit to the mid-low-state difference spectrum. Both plots are adjusted to the rest frame of $1 \mathrm{H} 0419-577$.

FIG. 9.-Top: pn camera data from the mid-low-flux state observation dicamera data from the mid-high-flux state observation divided by the data from the high-flux-state observation. 


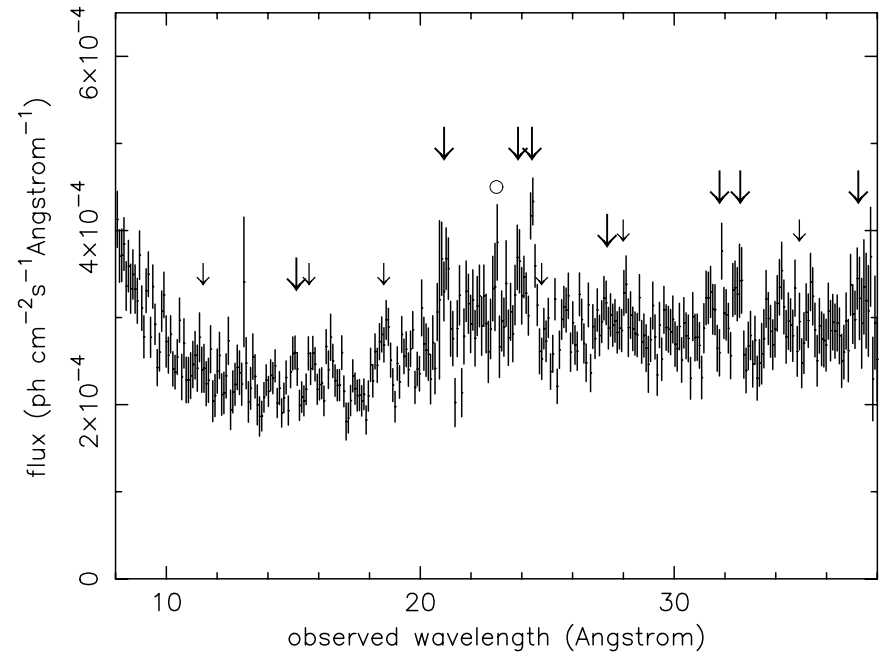

FIG. 11.-RGS spectrum of 1H 0419-577 summed over all five new $X M M$-Newton observations. The fluxed spectrum is binned at $85 \mathrm{~m} \AA$ resolution. Reading from left to right the larger arrows indicate the wavelengths of the principal candidate emission lines of Ne IX (f), O VIII Ly $\alpha, \mathrm{O}$ VII $(r, f)$, $\mathrm{N}$ VII $\operatorname{Ly} \alpha, \mathrm{N}$ VI $(r, f)$ and $\mathrm{C}$ VI $\operatorname{Ly} \alpha$, with the smaller arrows indicating the threshold wavelengths of the RRC of $\mathrm{Ne}$ IX, $\mathrm{O}$ VIII, $\mathrm{O}$ VII, $\mathrm{N}$ VI, $\mathrm{C}$ VI, and $\mathrm{C} \mathrm{v}$. The open circle at $\sim 23 \AA$ denotes a calibration defect.

XMM-Newton grating data of $1 \mathrm{H} 0419-577$, initially summing the data from all five orbits (512-690) to get the best statistics for what is a relatively faint source. Figure 11 displays the RGS-1 and RGS-2 fluxed spectrum, binned relatively coarsely at $85 \mathrm{~m} \AA$. The only spectral lines detected are all in emission, although the broad deficit between $\sim 10$ and $20 \AA$ is qualitatively consistent with the absorption trough seen in the EPIC spectra. The narrower absorption feature observed near $17.5 \AA$ is also consistent with the Fe 2-3 unresolved transition array (UTA; Behar et al. 2001) indicated in the XSTAR model fits (Fig. 10). The zero-velocity wavelengths of the principal K-shell emission lines and radiative recombination continua (RRC) falling in the $8-38 \AA$ waveband are indicated on the figure, and several are clearly detected.

To quantify the individual spectral features, we modeled the RGS data with a simple power law, with $\Gamma \sim 2.45$ yielding a reasonably good continuum fit $\left(\chi^{2}=4264 / 4084\right)$ over the 8-38 $\AA$ band, and then added Gaussians to each candidate emission line in turn, with wavelength, line width, and flux as free parameters. Four emission lines were formally detected, those of $\mathrm{O}$ VIII Ly $\alpha$, the resonance (r) and forbidden (f) lines of the $\mathrm{O}$ VII $1 s-2 p$ triplet, and the forbidden line of $\mathrm{N}$ VI, together with the RRC of $\mathrm{O}$ vII. Table 1 lists the results. Interestingly, the Gaussian line fittings support the visible impression from the fluxed spectra that the profiles of the resonance lines of $\mathrm{O}$ VII and $\mathrm{O}$ VIII are resolved, the best-fit line widths corresponding to a velocity width of $7000 \pm 3000 \mathrm{~km} \mathrm{~s}^{-1}$.
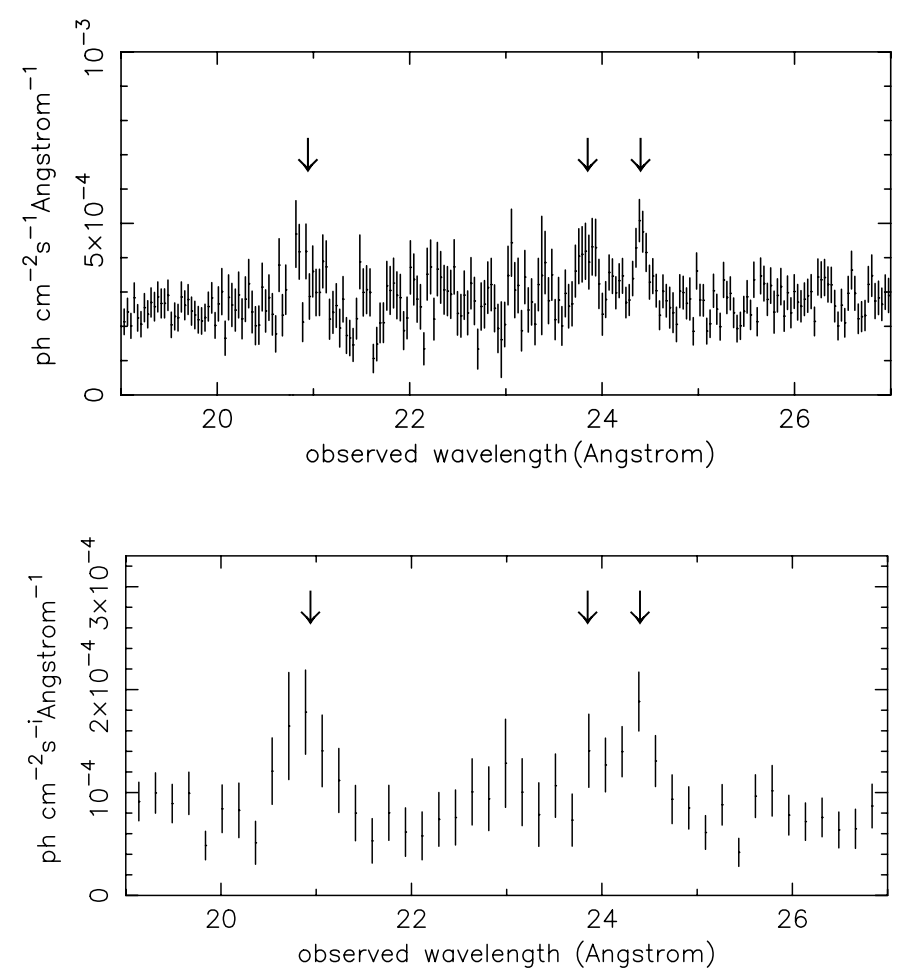

FIG. 12.-Top: Fluxed intermediate state RGS spectrum of 1H 0419-577 covering the wave band of $\mathrm{O}$ VIII $\mathrm{Ly} \alpha$ and the $\mathrm{O}$ VII $1 s-2 p$ triplet binned at $35 \mathrm{~m} \AA$ resolution. Bottom: The same spectral band from the low-flux state observation of $1 \mathrm{H} 0419-577$ binned at $170 \mathrm{~m} \AA$ resolution. The peak near $23 \AA$ is due to imperfect modeling of the O-K absorption edge in the detector response function.

Figure 12 (top) shows the $\mathrm{O}$ VII and $\mathrm{O}$ VIII lines at a higher resolution ( $35 \mathrm{~m} \AA$ bin width) and is compared in Figure 12 (bottom) with the same spectral region observed in the low-state spectrum. Because of the much poorer statistics the latter fluxed spectrum is more coarsely binned, at $170 \mathrm{~m} \AA$, and shows why only the $\mathrm{O}$ VIII Ly $\alpha$ and $\mathrm{O}$ VII (f) lines were identified in the earlier analysis of the low-state RGS spectrum (Paper I). The improved statistics of the full spectrum now allows the $\mathrm{O}$ VII (r) line to be resolved, although the total line fluxes remain consistent (within a factor $\sim 1.5$ ) with those observed in the lowflux state (Paper I). That consistency is significant in the context of our identification of a constant core soft emission ( $(9)$, since the observed overall $\sim 0.3-1 \mathrm{keV}$ flux, including the variable continuum component, varies by a factor $\sim 4$ over the five new XMM-Newton observations (Fig. 1).

\section{RECONCILING THE RGS AND EPIC SPECTRA}

The most important finding from our analysis of the EPIC spectra is of a substantial column density of low-ionization matter affecting the strongly variable emission component.

TABLE 1

Identified Emission Features in the RGS Spectrum

\begin{tabular}{|c|c|c|c|c|c|}
\hline Feature & $\begin{array}{l}\lambda \\
(\AA)\end{array}$ & $\begin{array}{l}\sigma / k T \\
(\mathrm{eV})\end{array}$ & $\begin{array}{c}\text { Flux } \\
\left(10^{-5} \text { photons } \mathrm{cm}^{-2} \mathrm{~s}^{-1}\right)\end{array}$ & $\begin{array}{l}\mathrm{EW} \\
(\mathrm{eV})\end{array}$ & $\Delta \chi^{2}$ \\
\hline 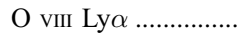 & 19.0 & $5 \pm 2$ & $6 \pm 2$ & $9 \pm 3$ & 22 \\
\hline $\mathrm{O}$ VII $1 s-2 p(\mathrm{r}) \ldots \ldots \ldots$ & 21.6 & $5 \pm 2$ & $6 \pm 2$ & $7 \pm 2.5$ & 22 \\
\hline $\mathrm{O}$ VII $1 s-2 p(\mathrm{f}) \ldots \ldots$. & 22.1 & $1 \pm 1$ & $4 \pm 1.5$ & $3.5 \pm 1.2$ & 32 \\
\hline $\mathrm{N}$ VI $1 s-2 p$ (f) $\ldots \ldots \ldots$ & 29.5 & $1 \pm 1$ & $5 \pm 3$ & $3 \pm 1.5$ & 14 \\
\hline O vII RRC ................ & 16.8 & $3.3 \pm 1.8$ & $1.9 \pm 1.1$ & $4 \pm 2$ & 18 \\
\hline
\end{tabular}




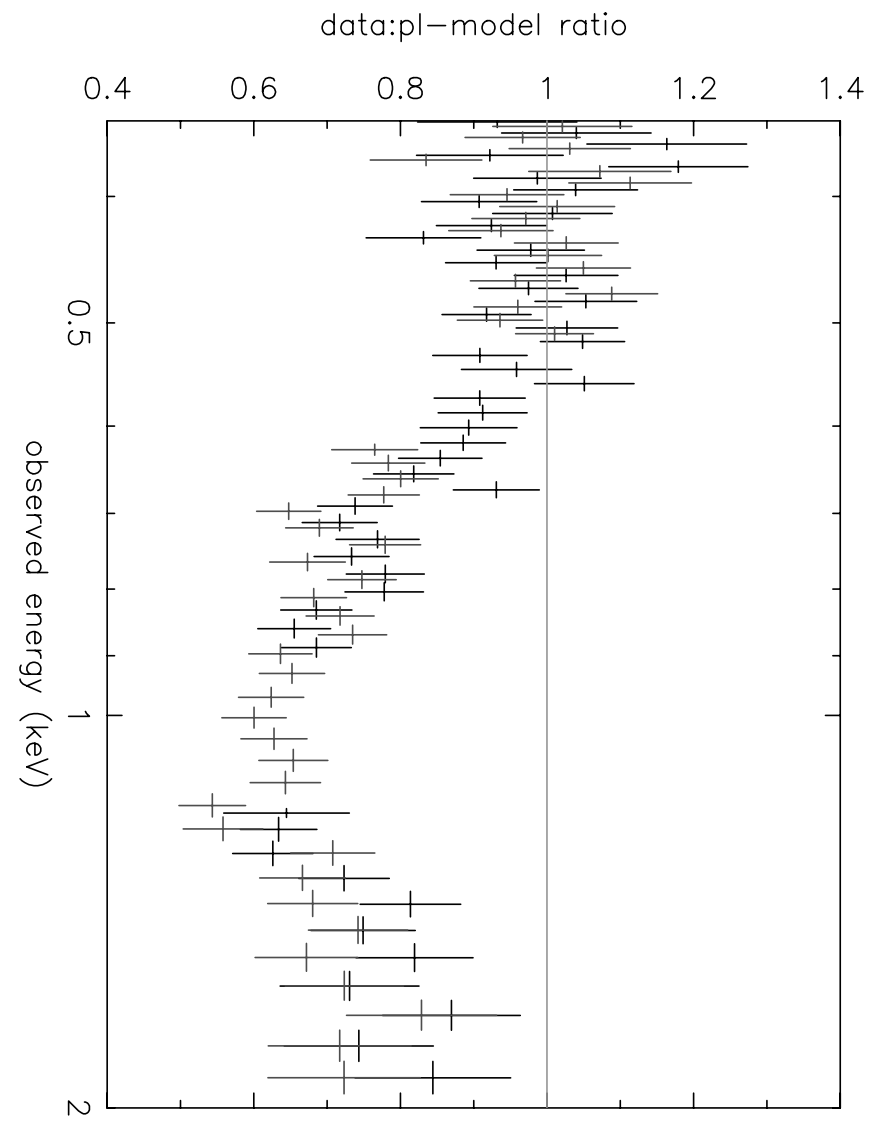

FIG. 13.-Coarsely binned RGS intermediate-state difference spectrum of $1 \mathrm{H} 0419-577$ plotted against a power law to illustrate absorption edge structures consistent with those seen in the simultaneous EPIC spectrum. [See the electronic edition of the Journal for a color version of this figure.]

At first sight, the RGS data seem to be in conflict with the above picture, since no narrow absorption features are observed, even though the EPIC ratio spectra (Fig. 9, bottom) show significant broadband absorption remaining up to the mid-high-flux state. However, we note that the RGS is designed to detect emission and absorption lines and is much less sensitive than EPIC in detecting continuum absorption. It is also possible that the absorbing material, if located close to the continuum emission region, is velocity broadened. In that context we note that the $\mathrm{O}$ VII and $\mathrm{O}$ VIII resonance emission lines are marginally resolved, suggesting an origin in moderately ionized matter with a velocity width of $7000 \pm 3000 \mathrm{~km}$ $\mathrm{s}^{-1}$, while it is conceivable that a still higher velocity or more turbulent outflow at smaller radii is responsible for the main absorption in the EPIC difference spectra. The width of the Fe K line in the intermediate-state spectrum $(\S 3)$ indicates such a higher velocity dispersion if interpreted as arising from fluorescence in the overlying absorber.

To check for comparable absorption edge structure in the RGS data we subtracted the low-state data from the summed spectra for 2003 March, June, and September, producing an intermediate-state RGS difference spectrum, which we plot in Figure 13 against a simple power-law model. With coarser binning (than in Fig. 11), the plot is consistent with absorption edges similar to those found in the XSTAR fits to the intermediate-state EPIC difference spectrum (Fig. 6, middle), although the statistics are not good enough to usefully constrain any velocity-broadened edges.

\section{A REAPPRAISAL OF THE SOFT X-RAY EXCESS}

Modeling the soft excess (conventionally defined as the excess soft X-ray flux above an extrapolation of the $2-10 \mathrm{keV}$ power law) with one or more blackbodies, as in $\S 3$, is a common practice in X-ray astronomy. However, since the implied blackbody temperatures are much higher than are appropriate for an AGN accretion disk, a common explanation is that the disk photons gain energy by electron scattering in a hotter "skin" or a corona lying above the disk. Nevertheless, such Comptonization models have remained rather ad hoc. Recently, a scaling by black hole mass of (more robust) Comptonization models for Galactic black hole sources (Done \& Gierlinski 2003) failed to explain the sharp upturn often seen below $\sim 1 \mathrm{keV}$ in AGN spectra. Noting also the similar shape (or blackbody temperature) of soft excess in AGNs over a wide luminosity range, those authors proposed that the soft excess in AGNs could be an artifact of "unseen or ignored" absorption (Gierlinski \& Done 2004).

The idea that absorption could be playing a larger part than that normally assumed in shaping the broadband spectra of Seyfert 1 galaxies was put forward in an early minisurvey of XMM-Newton observations by Pounds \& Reeves (2004). In Figure 1 of Pounds \& Reeves (2004) we showed the similarity in the observed broadband spectra of AGNs over a wide luminosity range, while noting that the more luminous sources exhibited a more gradual onset of the soft excess. In comparing the present EPIC spectra of 1H 0419-577 we now find the same qualitative trend with flux level for an individual AGN. Figure 14 compares the apparent soft excess above a $2-10 \mathrm{keV}$

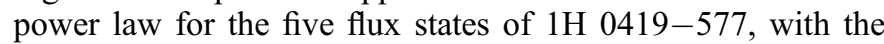
higher flux states showing a more gradual (or hotter) soft excess.

Our present analysis suggests that the "conventional" soft excess is indeed strongly affected by absorption. The additional point to emphasize here is that the individual difference spectra for high, intermediate, and mid-low states of $1 \mathrm{H} 0419-$ 577 exhibit no soft excess. Instead, we identify a core soft $X$-ray emission component in the extreme low-flux observation of September 2002 (Paper I). Thermal emission from the accretion disk modified by scattering in a hotter skin or corona and reflection from a hot inner disk surface are possible contributors to this core soft component. However, our present analysis of $1 \mathrm{H} 0419-577$, with evidence for a substantial column of cold nuclear gas becoming less opaque as the X-ray flux increases, suggests that recombination emission from associated photoionized (and possibly outflowing) matter is a natural origin of the soft excess. To test that idea we can compare the core soft excess observed in the XMM-Newton lowstate observation of $1 \mathrm{H} 0419-577$ with an emission model grid from XSTAR.

As with the absorption grids, the ionizing continuum is a power law of energy index -1 . The key variable parameters in this test were the ionization state and the relative metal abundances, which essentially determine the emission spectrum for comparison with the overall shape of low-state soft excess observed in EPIC and with the (few) strongest features identified in the RGS spectrum.

Figure 15 (top) shows the soft X-ray emission component, illustrated by removal of the blackbody and Gaussian emission line attributed to a blend of the O VII triplet and O VIII Ly $\alpha$ from the broadband fit to the low-flux state EPIC spectrum discussed in Paper I. Quantitatively, the X-ray flux in that soft excess $(0.3-1 \mathrm{keV})$ is $\sim 1.3 \times 10^{-12} \mathrm{ergs} \mathrm{s}^{-1}$, with some $30 \%$ of the flux removed by the low-energy absorption trough in 

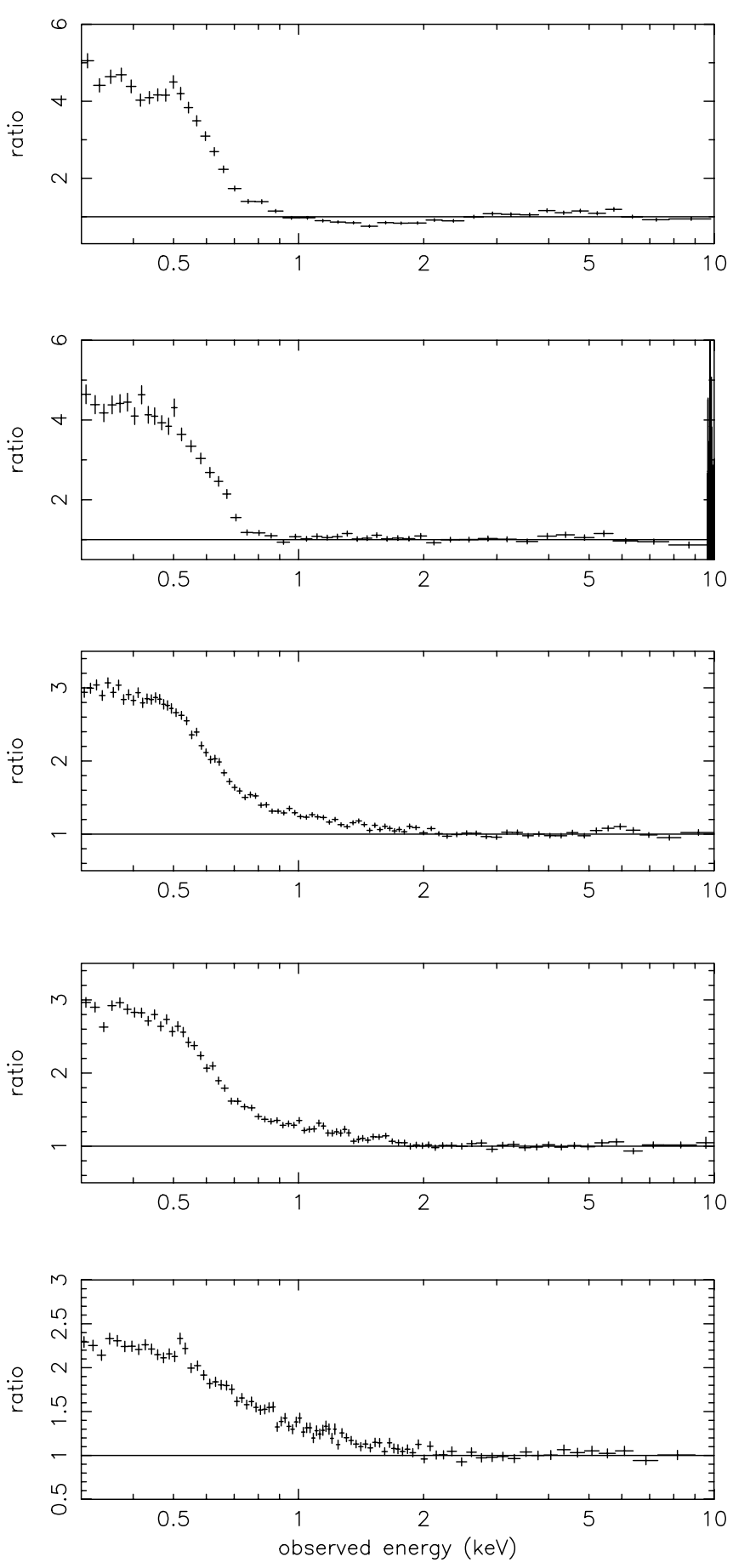

FIG. 14. - Soft excess above a $2-10 \mathrm{keV}$ power-law fit for, from top to bottom, the low-, mid-low-, intermediate-, mid-high-, and high-flux states of $1 \mathrm{H} 0419-577$. The respective $2-10 \mathrm{keV}$ photon indices are $1.07,1.32,1.61$, 1.69 , and 1.86 .

the intermediate-flux state (possibly the mean) spectrum of 1H 0419-577. Replacing the blackbody and O-K emission components with a grid of photoionized emission models in XSTAR produced a statistically good fit $\left(\chi^{2}=1028 / 1058\right.$ dof $)$; i.e., it was able to reproduce the shape of the core soft emission, with an ionization parameter $\log \xi \sim 1.3$ and element abundances for $\mathrm{C}, \mathrm{N}, \mathrm{O}, \mathrm{Ne}$, and $\mathrm{Fe}$ (relative to solar) of $0.4,0.4,0.15,0.35$, and 0.45 , respectively. We note that the ratio of the key elements, $\mathrm{O}$ and $\mathrm{Fe}$, suggests an overabundance of $\mathrm{Fe}$ similar to that found in the absorption modeling. Figure 15 (bottom) re-
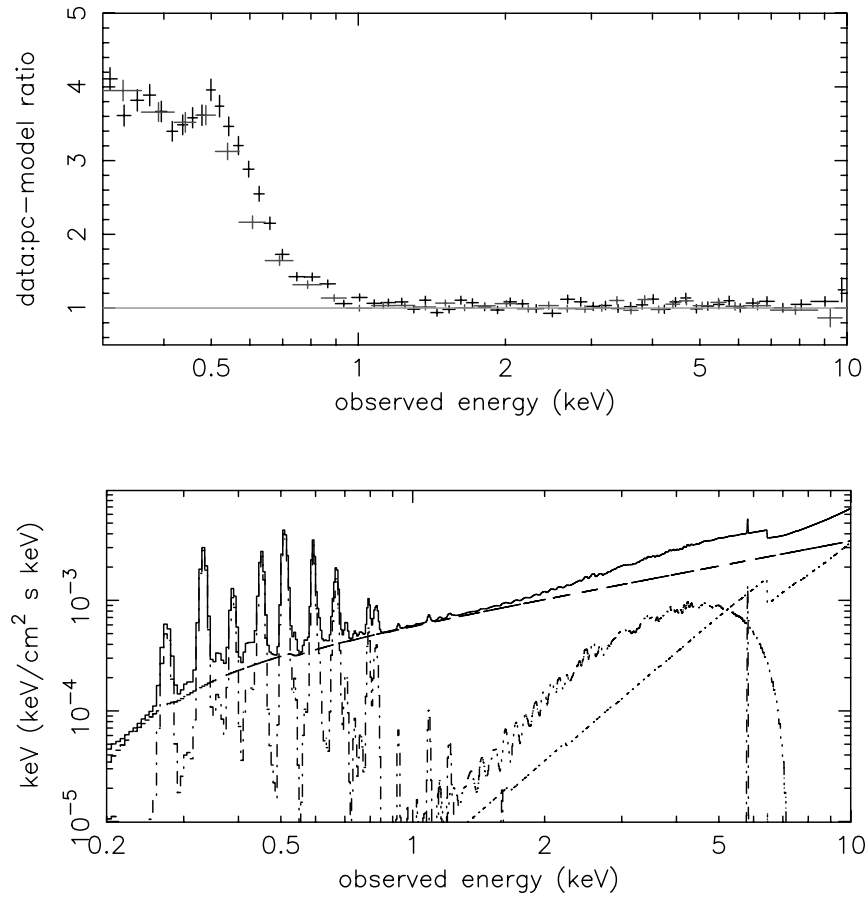

FIG. 15.-Top: Core soft excess obtained by subtracting the blackbody and $0.6 \mathrm{keV}$ Gaussian line components from the $0.3-10 \mathrm{keV}$ power law plus Laor (1991) line fit to the low-state EPIC data. Bottom: XSTAR emission spectrum replacing the blackbody and Gaussian line components in an alternative fit to the core soft excess. Details are given in $\S 9$. [See the electronic edition of the Journal for a color version of part (a) of this figure.]

produces the fitted XSTAR emission spectrum, showing how the emission profile, attenuated by the Galactic absorption in the line of sight to $1 \mathrm{H} 0419-577$, matches the strongly peaked soft excess in Figure 15 (top). It may also be understood, qualitatively, how the only lines detected in the full RGS spectrum of $1 \mathrm{H} 0419-577$, velocity broadened in the figure by a Gaussian width of $\sigma \sim 5 \mathrm{eV}$, are the principal emission lines of $\mathrm{O}$ VII, $\mathrm{O}$ VIII, $\mathrm{N}$ VI, and $\mathrm{C}$ VI.

Finally, it is interesting to compare the $\mathrm{Fe} \mathrm{K}$ line in the model of Figure 15 (bottom) with the emission line detected in the intermediate-flux state EPIC data (Figs. 2, top, and 3). Relative to the resonance lines of $\mathrm{O}$ VII and $\mathrm{O}$ VIII, the XSTAR model yields an Fe line that is a factor of $\sim 10$ weaker and centered at $\sim 6.42 \mathrm{keV}$ (corresponding to Fe XVII) compared with the EPIC line energy of $6.25 \pm 0.12 \mathrm{keV}$. It seems probable, therefore, that the bulk of the observed Fe K line arises by fluorescence in low-ionization matter, with the absorbing column being an obvious candidate. In that case, the observed line width and mean energy both indicate that the reprocessing matter lies within $\sim 100$ Schwarzschild radii of the SMBH, while the equivalent width requires a substantial covering factor.

\section{DISCUSSION}

The above analysis assumes that the underlying hard power law and soft excess seen in the low-flux state of $1 \mathrm{H} 0419-577$ in 2002 September remains constant (or, at least, much less strongly variable) over a timescale of $1-3$ yr. The similar powerlaw index/compTT parameters and the absence of a soft excess in the mid-low-, intermediate-, and high-flux state difference spectra support that assumption and in turn suggest that the core and variable X-ray emission components have a separate origin. 
Our good fortune in observing $1 \mathrm{H} 0419-577$ over a wide range of flux levels has yielded three main results. First, examination of the difference spectra of the intermediate- and mid-low-state EPIC data supports the conclusion in Paper I that the variable emission component has the form of a steep power law or cool Comptonized thermal spectrum. Second, this variable emission continuum shows the imprint at intermediateflux levels of absorption in low-ionization matter, the effect of which decreases as the source flux increases. Third, we note that the X-ray luminosity "lost" in the time-averaged absorbed flux is of the same order as that in the soft X-ray emission compo-

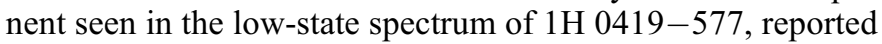
in Paper I, suggesting a possible origin of the core soft X-ray emission.

Thermal Comptonization remains a likely mechanism for the variable continuum emission component, with an excellent match to the high-state difference spectrum. We also have evidence from simultaneous measurements of $1 \mathrm{H} 0419-577$ with the Optical Monitor on XMM-Newton (Mason et al. 2001) that the UV $(2120,2910 \AA)$ flux increased by $\sim 20 \%$ from the lowto the intermediate-flux state (with smaller increases of $\sim 10 \%$ in the $U$ band and $\leq 5 \%$ in $V$ ), consistent with the larger change in the UV between the high- and low-flux states (Paper I). These data appear to confirm a link between the X-ray spectral change and enhanced thermal emission from the accretion disk. However, while increased disk emission could drive that change the similarity in the luminosity increase in the soft $\mathrm{X}$-ray and UV bands leaves open the alternative possibility that the UV flux increase is due to the reprocessing of soft X-rays directed toward the (unobscured) outer disk. In the latter case, the brightening X-ray continuum would primarily be a consequence of an increased optical depth of Comptonizing electrons. We note in passing that if the scenario we propose for $1 \mathrm{H} 0419-577$ is widely applicable to radio-quiet AGNs, then the hard (possibly disk-corona emission) and softer continuum components must be coupled in some way in order to explain the canonical power-law index (neglecting reflection) of $\Gamma \sim$ 1.7 at $2-10 \mathrm{keV}$, observed in broadband fits for Seyfert 1 galaxies in general (Nandra \& Pounds 1994), as well as the longterm average spectrum of $1 \mathrm{H} 0419-577$.

Given that the peak luminosity of the variable X-ray emission component is $\leq 10 \%$ of the bolometric (accretion) luminosity of $1 \mathrm{H} 0419-577$, the kinetic energy in a wind could be sufficient to support a separate emission process. Although we have found no unambiguous evidence for an outflow in 1H 0419-577, the resolved emission-line profiles of Fe K, $\mathrm{O}$ VII, and $\mathrm{O}$ VIII imply a large velocity dispersion, while it seems likely that as the cold absorber is photoionized some fraction will be driven away by the strong continuum radiation pressure. We also note the growing evidence in other recent studies for ionized outflows at velocities of $\sim 5 \%-20 \%$ of $c$ (Chartas et al. 2002, 2003; Pounds et al. 2003, 2004a; Reeves et al. 2003). Any such outflow is likely to have an initial velocity that reflects the gravity of its origin (as with stellar winds), so that a wind emerging from a small radius will have a correspondingly high velocity. The kinetic energy carried by such a "black hole wind" (King \& Pounds 2003) could then support a separate emission mechanism of order $v / c$ times the bolometric luminosity, with shocks in the outflow (e.g., Camenzind \& Courvoisier 1983) perhaps providing the enhanced Comptonizing electrons. A timescale for major spectral change in $1 \mathrm{H} 0419-577$ being on the order of several months, one possible cause of an increased outflow may be the coalignment of magnetic field lines in the inner disk (Livio et al. 2003). Alternatively, if the variable $\mathrm{X}$-ray emission arises from the base of a jet (e.g., Markoff et al. 2001) then it is interesting to note that synchrotron radiation could, qualitatively, also explain the spectral form of 1H 0419-577.

Absorption features in both the difference and flux-ratio spectra of 1H 0419-577 provide clear evidence for substantial low-ionization matter overlying (or embedded in) the X-ray continuum emission region. The marked decrease in opacity and/or covering factor of the absorber as the X-ray flux increases indicates that the absorbing matter lies close to the SMBH, a conclusion also consistent with the Seyfert 1 classification of $1 \mathrm{H} 0419-577$. The low-ionization parameter then requires the absorbing matter to be of high density $\left(\gtrsim 10^{17} \mathrm{~cm}^{-3}\right)$ to survive the intense continuum irradiation. A possible scenario is for the absorbing matter to be in small, dense clouds that partially cover the continuum source (e.g., Ferland \& Rees 1988), the covering factor decreasing as the source flux increases, a change modeled by a reducing column density in our spectral fitting. Dense matter at the base of a jet or outflow provides other potential sites. The recent discussion of "aborted jets," where dense blobs of matter are ejected from the inner accretion disk at subescape velocities (Ghisellini et al. 2004), also offers a geometry that might be compatible with the results we report here.

Although the spectral variability in $1 \mathrm{H} 0419-577$ is extreme, the low-flux state spectrum is remarkably similar in appearance to the low-state spectra of two other bright Seyfert 1 galaxies, NGC 4051 (Pounds et al. 2004a; Uttley et al. 2004) and MCG -6-30-15 (e.g., Reynolds et al. 2004). In each case a hard power-law continuum also exhibits a broad spectral feature at $\sim 3-6 \mathrm{keV}$ that may alternatively be fitted by an extreme relativistic Fe $\mathrm{K}$ line or by partial covering of the X-ray continuum by low-ionization matter. In the disk-corona model of hard X-ray emission, where UV photons from the accretion disk are upscattered in an overlying corona (e.g., Haardt \& Maraschi 1991), an unusually hard spectrum indicates a relatively low UV flux and "photon-starved" corona. The intrinsic hardness of the core spectrum would be reduced somewhat if continuum reflection is enhanced, for example, by light bending in the strong gravity near the black hole, as proposed by Miniutti \& Fabian (2004) to explain the extreme $\mathrm{Fe} \mathrm{K}$ emission line in the low-state spectrum of MCG -6-30-15. In that case a simultaneous observation by BeppoSAX supported the enhanced reflection rather than partial covering models. However, in the light of the new evidence reported here for substantial cold matter close to the black hole in $1 \mathrm{H} 0419-577$ it is hard to exclude the possibility that absorption also modifies the strength and profile of the key diagnostic $\mathrm{Fe} \mathrm{K}$ emission line in AGN spectra.

The strong forbidden line of $\mathrm{O}$ VII is evidence that a substantial part of the core soft X-ray emission comes from an ionized gas of relatively low density, where the emission measure then yields a radial extent (Paper I) sufficient to maintain the soft X-ray emission as essentially constant over the $3 \mathrm{yr}$ of our XMM-Newton studies of 1H 0419-577. The detection of resolved resonance lines of $\mathrm{O}$ VII and $\mathrm{O}$ VIII and a strong RRC of $\mathrm{O}$ VII in the full RGS spectrum suggests an additional component to the soft X-ray emission from higher density, and perhaps turbulent, recombining gas closer to the continuum source. The comparable luminosity of the core soft X-ray emission with that of the absorbed continuum in the intermediate (average) flux state then offers a natural explanation of the soft X-ray emission, when an ionized outflow subsequently recombines. The "true" soft excess in Seyfert 1 galaxies, as we find for $1 \mathrm{H} 0419-577$, may then be more akin to the soft X-ray 
emission seen in Seyfert 2 galaxies arising from an extended region of ionized gas.

Finally, we point out that the detection of cold absorbing matter close to the SMBH in a luminous Seyfert 1 galaxy implies an additional component to the standard model by which type 1 and type 2 AGNs are distinguished in relation to absorption in a distant torus (Antonucci 1993).

\section{SUMMARY}

1. A series of XMM-Newton observations of the luminous Seyfert 1 galaxy 1H 0419-577 have shown that the large-scale spectral variability is primarily due to a steep power law or cool Comptonized thermal emission component. In seeking an alternative to the disk-corona model for this new emission component it is interesting to note that mechanical energy in a wind could be sufficient to support such a process, perhaps via shocks in a turbulent or inhomogeneous outflow.

2. Broad absorption features superposed on the variable emission continuum require substantial cold, dense matter apparently lying close to the central SMBH. As 1H 0419-577 brightens from a low- to a high-flux state, the ionization parameter of this absorbing matter increases and its column density (or covering factor) falls.

3. An underlying assumption in our analysis is that a hard core spectral component remains essentially unchanged over the 1-3 yr period of the observations. We note the similarity of this core X-ray spectrum of $1 \mathrm{H} 0419-577$ to high-quality lowstate spectra of other Seyfert 1 galaxies, including NGC 4051 and MCG $-6-30-15$, which also show a flat power law, rela- tivistic Fe K emission line or partial covering, and strong "soft excess."

4. In the case of $1 \mathrm{H} 0419-577$, we find that the variable soft excess is essentially an artifact of continuum absorption, while a core soft X-ray emission component has a spectral form and luminosity consistent with the re-emission of the absorbed $\mathrm{X}$-ray continuum in an extended region of ionized gas.

5. While the X-ray spectral variability of $1 \mathrm{H} 0419-577$ is extreme, its explanation in terms of a variable soft emission component, modified by absorption in low-ionization matter close to the SMBH, is unlikely to be unique, suggesting the need for reappraisal of other high-quality AGN X-ray spectra.

6 . Finally, we note that the occurrence of a substantial column density of cold gas overlying the hard X-ray source in a luminous AGN has implications both for the diagnostic potential of the $\mathrm{Fe} \mathrm{K}$ emission line and the new source population required to explain the hard ( $\gtrsim 5 \mathrm{keV})$ cosmic X-ray background spectrum.

The results reported here are based on observations obtained with XMM-Newton, an ESA science mission with instruments and contributions directly funded by ESA member states and the US (NASA). The authors wish to thank the SOC and SSC teams for organizing the XMM-Newton observations and initial data reduction, Tim Kallman for provision of a new XSTAR grid, and the referee for constructive comments on the initial text. K. A. P. is pleased to acknowledge a Leverhulme Trust Emeritus Fellowship.
Antonucci, R. R. J. 1993, ARA\&A, 31, 473

Arnaud, K. A. 1996, in ASP Conf. Ser. 101, Astronomical Data Analysis Software and Systems V, ed. G. H. Jacoby \& J. Barnes (San Francisco: ASP), 17

Behar, E., Sako, M., \& Kahn, S. M. 2001, ApJ, 563, 497

Brissenden, R. J. V., Tuohy, I. R., Remillard, R. A., Buckley, D. A., Bicknell, G. V, Bradt, H. V., \& Schwartz, D. A. 1987, Proc. Astron. Soc. Australia, 7, 212

Camenzind, M., \& Courvoisier, T. 1983, ApJ, 266, L83

Chartas, G., Brandt, W. N., \& Gallagher, S. C. 2003, ApJ, 595, 85

Chartas, G., Brandt, W. N., Gallagher, S. C., \& Garmire, G. P. 2002, ApJ, 579, 169

den Herder, J. W., et al. 2001, A\&A, 365, L7

Done, C., \& Gierlinski, M. 2003, MNRAS, 342, 1041

Fabian, A. C., \& Vaughan, S. 2003, MNRAS, 340, L28

Ferland, G. J., \& Rees, M. J. 1988, ApJ, 332, 141

Ghisellini, G., Haardt, F., \& Matt, G. 2004, A\&A, 413, 535

Gierlinski, M., \& Done, C. 2004, MNRAS, 349, L7

Grupe, D., Wills, B. J., Leighly, K. M., \& Meusinger, H. 2004, AJ, 127, 156

Guainazzi, M., et al. 1998, A\&A, 339, 327

Haardt, F., \& Maraschi, L. 1991, ApJ, 380, L51

Kallman, T., \& Bautista, M. 2001, ApJS, 133, 221

Kallman, T., Liedahl, D., Osterheld, A., Goldstein, W., \& Kahn, S. 1996, ApJ, 465, 994

Kaspi, S., Smith, P. S., Netzer, H., Maoz, D., Jannuzi, B. T., \& Giveon, U. 2000, ApJ, 533, 631

King, A. R., \& Pounds, K. A. 2003, MNRAS, 345, 657

\section{REFERENCES}

Laor, A. 1991, ApJ, 376, 90

Livio, M., Pringle, J. E., \& King, A. R. 2003, ApJ, 593, 184

Markoff, S., Falcke, H., \& Fender, R. 2001, A\&A, 372, L25

Mason, K. O., et al. 2001, A\&A, 365, L36

Miniutti, G., \& Fabian, A. C. 2004, MNRAS, 349, 1435

Nandra, K., \& Pounds, K. A. 1994, MNRAS, 268, 405

Nelson, C. H. 2000, ApJ, 544, L91

Page, K., Pounds, K. A., Reeves, J. N., \& O’Brien, P. 2002, MNRAS, 330, L1

Pounds, K. A., \& Reeves, J. N. 2004, in New Visions of the X-Ray Universe in the XMM-Newton and Chandra Era, ed. F. Jansen (ESA SP-488; Noordwijk: ESA), in press (astro-ph/0201426)

Pounds, K. A., Reeves, J. N., King, A. R., \& Page, K. L. 2004a, MNRAS, 350, 10

Pounds, K. A., Reeves, J. N., King, A. R., Page, K. L., O’Brien, P. T., \& Turner, M. J. L. 2003, MNRAS, 345, 705

Pounds, K. A., Reeves, J. N., Page, K. L., \& O’Brien, P. T. 2004b, ApJ, 605, 670 (Paper I)

Reeves, J. N., O’Brien, P. T., \& Ward, M. J. 2003, ApJ, 593, L65

Reynolds, C. S., Wilms, J., Begelman, M. C., Staubert, R., \& Kendziorra, E. 2004, MNRAS, 349, 1153

Shih, D. C., Iwasawa, K., \& Fabian, A. C. 2002, MNRAS, 333, 687

Strüder, L., et al. 2001, A\&A, 365, L18

Titarchuk, L. 1994, ApJ, 434, 570

Turner, M. J. L., et al. 2001, A\&A, 365, L27

Turner, T. J., et al. 1999, ApJ, 510, 178

Uttley, P., Taylor, R. D., McHardy, I. M., Page, M. J., Mason, K. O., Lamer, G., \& Fruscione, A. 2004, MNRAS, 347, 1345 